\title{
Clinical Features of Strabismus in Patients with Congenital Optic Disc Anomaly
}

\author{
Hyeshin Jeon ${ }^{1,2}$, Yeonji Jo ${ }^{1,2}$, Hee-young Choi ${ }^{1,2}$ \\ ${ }^{1}$ Department of Ophthalmology, Pusan National University School of Medicine, Busan, Korea \\ ${ }^{2}$ Medical Research Institute, Pusan National University Hospital, Busan, Korea
}

Purpose: To investigate the clinical features of strabismus in patients with congenital optic disc anomaly and compare and analyze the characteristics of patients who showed changes in the strabismus pattern with those who did not.

Methods: Medical records of the patients who were diagnosed with both strabismus and congenital optic disc anomaly and followed-up for $\geq 1$ year were reviewed retrospectively. Clinical characteristics and ophthalmic features at the initial visit and final follow-up were assessed. Patients with a change in the direction of strabismus or a difference of $>10$ prism diopters in the deviation angle during the follow-up period were allocated to the changed group. The remaining patients were assigned to the unchanged group. The clinical characteristics of the two groups were compared.

Results: Twenty-eight patients (15 boys) were included (mean age, 39.0 months; range, 5-150 months). Three (10.7\%) patients were born preterm and four (14.3\%) had other underlying systemic disease. Sixteen (57.1\%) patients had exotropia, and 12 (42.9\%) had esotropia. Concurrent vertical strabismus was present in three (10.7\%) patients. Strabismus features changed in 14 (50.0\%, changed group) patients and remained unchanged in 14 (50.0\%, unchanged group) patients. Age, sex, and laterality did not differ between groups. Preterm birth history $(n=3)$ and combined systemic disease $(n=4)$ were only observed in the changed group ( $p=0.111$ and $p=0.049$, respectively).

Conclusions: Considering the possibility of changes in strabismic features, close monitoring of patients with strabismus combined with congenital disc anomaly is essential, particularly in those with preterm birth history or underlying systemic conditions.

Key Word: Amblyopia, Esotropia, Exotropia, Optic disc, Strabismus

Congenital optic disc anomalies underlie cases of decreased visual acuity, strabismus, and nystagmus in child-

Received: November 6, 2020 Final revision: December 22, 2020

Accepted: January 3, 2021

Corresponding Author: Hee-young Choi, MD, PhD. Department of Ophthalmology, Pusan National University School of Medicine, 179 Gudeokro, Seo-gu, Busan 49241, Korea. Tel: 82-51-240-7324, Fax: 82-51-2427341,E-mail: hychoi@pusan.ac.kr hood. These include optic nerve hypoplasia, excavated optic disc anomalies including morning glory syndrome, coloboma, tilted disc, and myelinated nerve fibers [1]. In addition, such anomalies are a possible cause of superimposed amblyopia and strabismus due to sensory deprivation, particularly in cases of unilateral structural abnormality [2].

Congenital optic disc anomaly accompanies strabismus in $53 \%$ to $71 \%$ of patients [3-5]. The high prevalence of 
strabismus and the risk of low visual acuity necessitates early detection and proper management to facilitate the development of the visual function. Strabismus surgery can also be considered. Some studies have shown that strabismus is associated with congenital optic nerve abnormalities, but studies on longitudinal changes of strabismus are lacking.

In this study, we aimed to investigate the clinical characteristics of strabismus in patients diagnosed with congenital optic disc anomalies from a longitudinal perspective. We further aimed to evaluate the characteristics of patients who experienced changes in the features of strabismus, following its conservative management during the study period.

\section{Materials and Methods}

\section{Ethics statement}

The institutional review board in Pusan National University Hospital (2103-002-100) approved this study and the procedures used in this study conformed to the principles of the Declaration of Helsinki. Given the retrospective nature of this study, the need for informed consent was waived.

\section{Patients' selection}

The medical records of patients aged $<14$ years who were diagnosed with both strabismus (horizontal strabismus $\geq 10$ prism diopters $[\mathrm{PD}]$ or any vertical strabismus) and congenital optic disc anomaly at our hospital between January 2005 and May 2019 and followed up for $\geq 1$ year were retrospectively reviewed. Each patient underwent a proper systemic workup, including radiological imaging or laboratory tests related to their congenital disc anomaly.

\section{Ophthalmic examination}

Slit-lamp and fundus examinations were performed to assess any combined ocular anatomical abnormalities. The refractive error, as determined using cycloplegic refraction (three drops of $1 \%$ cyclopentolate hydrochloride in each eye, with retinoscopy, conducted $30 \mathrm{~min}$ after administration of the initial drop), was recorded as spherical equiva- lent (SE) values. Significant refractive error included hyperopia (SE $\geq+1.5$ diopters $[\mathrm{D}]$ ), myopia ( $\mathrm{SE} \leq-1.5 \mathrm{D}$ ), high astigmatism (Dcyl $\geq 3.0 \mathrm{D})$ in at least one eye, and anisometropia (SE difference between both eyes $\geq 2.0 \mathrm{D}$ ). Visual acuity was measured using a Snellen visual acuity chart in cooperative children and was converted to logarithm of the minimum angle of resolution. Poor visual acuity, which cannot be represented by numbers, was converted as follows: counting fingers, 2; hand motion, 3 ; light perception, 4; and non-light perception, 5 [6]. The deviation angle was measured using a prism and alternate cover test. The Krimsky test was used in cases of non-cooperative or poor-fixating patients. Duction and version were also assessed. Refractive errors were corrected, and occlusion therapy was applied in cases of amblyopia.

The patients' clinical information, including sex, age, birth history, and systemic disease, and ophthalmic features, including the type of strabismus, deviation angle, abnormal ocular movement, refractive error, and visual acuity, at the initial visit and final follow-up were assessed. In patients who underwent strabismus surgery, the record immediately before surgery was used as the final follow-up observation. Since the children were often young and unable to cooperate at the time of recruitment, the initial visual acuity measurements during the observation period were analyzed separately.

\section{Statistical analysis}

Patients with a change in the direction of the strabismus or a change $>10 \mathrm{PD}$ in the deviation angle from the initial to the final presentation were allocated to the changed group. The remaining patients were assigned to the unchanged group. Fisher exact test and the Mann-Whitney $U$-test were used to compare the two groups. IBM SPSS Statistics ver. 21.0 (IBM Corp., Armonk, NY, USA) was used for all statistical analyses, and $p$-values $\leq 0.05$ were considered statistically significant.

\section{Results}

A total of 28 patients ( 15 boys and 13 girls) were included in the study. The mean age was 39.0 months (range, $5-150$ months). Twenty-three (82.2\%) patients had unilateral optic disc anomalies and five (17.9\%) had bilateral disc 


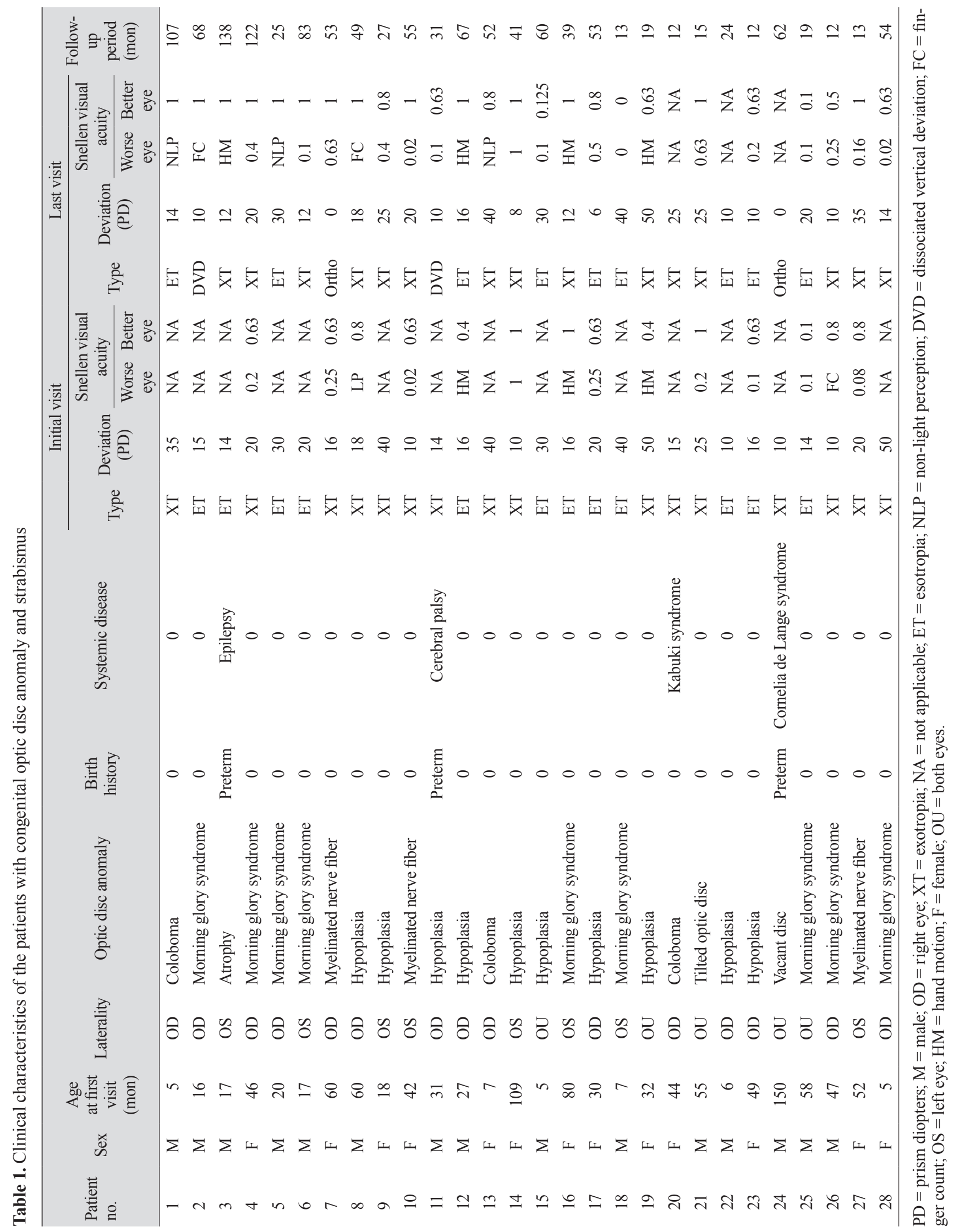


Table 2. Clinical features of the patients in the two groups according to the change of strabismus

\begin{tabular}{|c|c|c|c|}
\hline & Unchanged $(n=14)$ & Changed $(n=14)$ & $p$-value \\
\hline Age (mon) & $37.5(5-109)$ & $40.4(5-150)$ & 0.839 \\
\hline Sex (male : female) & $9: 5$ & $6: 8$ & 0.225 \\
\hline Laterality & & & 0.163 \\
\hline Unilateral & $10(71.4)$ & $13(92.9)$ & \\
\hline Bilateral & $4(28.6)$ & $1(7.1)$ & \\
\hline \multicolumn{4}{|l|}{ Optic disc pathology } \\
\hline Optic nerve hypoplasia & $7(50.0)$ & $3(21.4)$ & \\
\hline Morning glory syndrome & $5(35.5)$ & $4(28.6)$ & \\
\hline Coloboma & $1(7.1)$ & $2(14.2)$ & \\
\hline Myelinated nerve fiber & $0(0)$ & $3(21.4)$ & \\
\hline Optic disc atrophy & $0(0)$ & $1(7.1)$ & \\
\hline Tilted optic disc & $1(7.1)$ & $0(0)$ & \\
\hline Vacant disc & $0(0)$ & $1(7.1)$ & \\
\hline Preterm birth & $0(0)$ & $3(21.4)$ & 0.111 \\
\hline Systemic disease & $0(0)$ & $4(28.6)$ & 0.049 \\
\hline \multicolumn{4}{|l|}{ Refractive error } \\
\hline Worse eye (SE) & $-0.705(-7.0-+9.3)$ & $-1.43(-10.4-+13.9)$ & 0.667 \\
\hline Better eye (SE) & $0.429(-4.75-+5.75)$ & $0.723(-1.25-+4.25)$ & 0.946 \\
\hline Type of strabismus & & & 0.352 \\
\hline Exotropia & $7(50)$ & $9(64.3)$ & \\
\hline Esotropia & $7(50)$ & $5(35.7)$ & \\
\hline Combined vertical strabismus & $1(7.1)$ & $2(14.3)$ & 0.500 \\
\hline Ocular deviation at initial visit (PD) & $23.36(10-50)$ & $21.07(10-50)$ & 0.667 \\
\hline \multicolumn{4}{|l|}{ Visual acuity at final visit } \\
\hline Worse eye (logMAR) & $1.83(0-5.0)$ & $1.67(0.2-5)$ & 0.932 \\
\hline Better eye (logMAR) & $0.23(0-1.0)$ & $0.05(0-0.2)$ & 0.291 \\
\hline Follow-up period (mon) & $37.8(12-122)$ & $46.7(12-138)$ & 0.104 \\
\hline
\end{tabular}

Values are presented as mean (range), number, or number (\%); Change of strabismus was defined as change in the direction of the strabismus or a change of $>10$ PD in the deviation angle comparing the initial and final presentation.

$\mathrm{SE}=$ spherical equivalent; $\mathrm{PD}=$ prism diopters; $\log \mathrm{MAR}=$ logarithm of the minimum angle of resolution.

anomalies. The diagnosis of the anomaly was optic nerve hypoplasia in ten $(35.7 \%)$ patients, morning glory syndrome in nine $(32.1 \%)$, coloboma in three $(10.7 \%)$, myelinated nerve fiber in three (10.7\%), optic disc atrophy in one $(3.6 \%)$, tilted optic disc in one (3.6\%), and vacant disc in one $(3.6 \%)$. Three $(10.7 \%)$ patients were born preterm, and four $(14.3 \%)$ had other underlying systemic diseases, including epilepsy, cerebral palsy, Kabuki syndrome, and Cornelia de Lange syndrome (all $\mathrm{n}=1$ ).

Myopia, which was observed in 14 (50.0\%) patients, was the most common significant refractive error. Hyperopia was observed in six (21.4\%) patients, high astigmatism was observed in one patient (3.6\%), and anisometropia was observed in $11(39.3 \%)$ patients. The mean visual acuity of the worse eye was 1.60 (range, $0-4$ ), which was initially assessed in 14 patients. During the follow-up observations, visual acuity measurements were available for 24 patients; eight $(28.6 \%)$ of these patients presented with increased visual acuity compared to the initial presentation.

Sixteen (57.1\%) patients had exotropia and 12 (42.9\%) had esotropia. Concurrent vertical and horizontal strabismus was observed in three (10.7\%) patients. The clinical 
characteristics of the patients are presented in Table 1.

Strabismus features changed in 14 (50.0\%, changed group) patients, and remained unchanged in 14 (50.0\%, unchanged group). The direction changed in six (42.9\%) patients. Esotropia changed to exotropia in three patients and dissociated vertical deviation in one patient. Exotropia changed to esotropia in one patient and dissociated vertical deviation in one patient. The deviation angle changed in eight (57.1\%) patients. Esotropia decreased in one patient. Exotropia increased in three patients and decreased in four patients. Age, sex, and laterality did not differ between the two groups. Preterm birth history and combined systemic disease were only observed in the changed group $(p=0.111$ and $p=0.049$, respectively). Ophthalmologic characteristics, including refractive error, type, and degree of strabismus, and final visual acuity did not differ between the groups. The follow-up period was 37.8 months (range, 12122 months) in the unchanged group and 46.7 months (range, 12-138 months) in the changed group and did not significantly differ between the groups (Table 2).

\section{Discussion}

In our study, optic nerve hypoplasia was the most common congenital optic disc anomaly, and myopia was the most common refractive error. Overall, changes in strabismus features were observed in $50 \%$ of the patients. While there was no difference in ophthalmic characteristics among these patients, all patients with a history of preterm birth or systemic disease exhibited features of changed strabismus.

Similar frequencies of exotropia and esotropia were observed. Both exotropia and esotropia are predominantly observed in patients with congenital optic disc anomalies $[3,4,7,8]$. Myopia was the most common refractive error in our study. There are several previous reports of refractive errors in patients with congenital optic disc anomalies $[4,5,7,9]$. The diagnosis was heterogeneous and the rate of specific refractive error of each diagnosis was not analyzed individually because we recruited patients with both congenital optic disc anomaly and strabismus. This may explain the diversity of previous reports of refractive errors. Nevertheless, the high frequency of significant refractive errors and anisometropia suggests that proper refractive inspection and vigorous correction of refractive errors are necessary.

Twenty-nine percent of patients exhibited improvement in visual acuity from the first visual acuity measurement to that at the final follow-up, and the remaining patients exhibited no increase in visual acuity despite amblyopia treatment. This may be considered a poor prognosis but also highlights the possibility of enhancement of visual function in certain patients. A congenital optic disc anomaly can be superimposed with functional amblyopia [2]. Even if the amblyopic eye had structural abnormalities, amblyopia treatment could achieve increased visual acuity [10].

The angle of strabismus increased or decreased, and the direction of strabismus also changed, which appeared in various ways. There are some previous reports about the progression of strabismus [11,12]. VanderVeen et al. [12] reported that strabismus observed in high-risk retinopathy of prematurity patients disappeared within 1 year of age in $30 \%$ of patients. They also suggested that stability of ocular alignment could be delayed, owing to either ocular or developmental abnormalities as the cause. Similarly, younger age at the initial visit was strongly associated with an increased probability of spontaneous resolution of esotropia in a PEDIG study. The authors argued that this correlation is related to the immaturity of the ocular motor system [13]. It can be presumed that the change in clinical features of strabismus may be caused by the natural course of strabismus itself over time or related to changes in visual function following the treatment of amblyopia.

It is not uncommon for congenital optic nerve malformations to be accompanied by ophthalmic abnormalities or systemic diseases. Furthermore, Brodsky [14] noted that the subclassification of various forms of colobomatous defects has been updated to predict the likelihood of related central nervous system abnormalities based on the appearance of the optic disc. A previous report found that developmental delay (32\%), cerebral palsy $(13 \%)$, and seizures $(12 \%)$ were present in combination with optic nerve hypoplasia [4]. Mental-motor retardation, cerebral palsy, hydrocephalus, and epilepsy were also reported in $42.9 \%$ of patients with bilateral optic disc pathology and strabismus [7]. This study differed from our study in that it included both acquired optic disc atrophy and congenital optic nerve disease, but a high frequency of systemic disease suggests that a multidisciplinary approach is needed in these patients.

We included a patient with optic disc atrophy. Both optic 
nerve hypoplasia and optic atrophy could present during the gestational period, depending on the timing of the insult [15]. Since the patient was preterm, had epilepsy as a combined systemic disease, and had no other relevant secondary disease that could result in optic disc atrophy after birth, optic disc atrophy was considered a congenital deficit in this patient.

Our study has some limitations. First, as a retrospective study, the duration of follow-up was not consistent among patients. Second, because the number of patients was small, a detailed analysis of each optic nerve anomaly was not possible. A further large-scale study could overcome these limitations and add significance to this paper.

A significant number of patients exhibited clinical features of strabismus, and both the deviation angle and the direction of the strabismus changed either in the amount or the direction of the ocular deviation. Some patients exhibited improved visual acuity during the follow-up period. This suggests that the diagnosis and treatment of strabismus should be carefully determined in these patients. In addition, this study emphasizes that the changes are distinct among patients with systemic disease. Patients with accompanying systemic disease are often less cooperative, and therefore, ophthalmic evaluation is often delayed or forgone. However, active evaluation and treatment without omission should be considered.

In conclusion, careful monitoring is essential during identification and management of patients with strabismus accompanied by congenital optic nerve anomalies, considering the possibility of a significant change in the clinical features of strabismus, particularly in cases of a history of preterm birth or accompanying systemic disease.

\section{Conflict of Interest}

No potential conflict of interest relevant to this article was reported.

\section{Acknowledgements}

This work was supported by a 2-year research grant from Pusan National University 2019.

\section{References}

1. Brodsky MC. Pediatric neuro-ophthalmology. New York: Springer; 2010. p. 75-109.

2. Kushner BJ. Functional amblyopia associated with abnormalities of the optic nerve. Arch Ophthalmol 1984;102:683-5.

3. Kim MR, Park SE, Oh SY. Clinical feature analysis of congenital optic nerve abnormalities. Jpn J Ophthalmol 2006;50:250-5

4. Garcia ML, Ty EB, Taban M, et al. Systemic and ocular findings in 100 patients with optic nerve hypoplasia. $J$ Child Neurol 2006;21:949-56.

5. Berk AT, Yaman A, Saatci AO. Ocular and systemic findings associated with optic disc colobomas. $J$ Pediatr Ophthalmol Strabismus 2003;40:272-8.

6. Scanlon PH, Foy C, Chen FK. Visual acuity measurement and ocular co-morbidity in diabetic retinopathy screening. Br J Ophthalmol 2008;92:775-8.

7. Erkan Turan K, Taylan Sekeroglu H, et al. Bilateral optic disc pathologies as an accompanying feature of comitant strabismus in children. Int Ophthalmol 2018;38:425-8.

8. Siatkowski RM, Sanchez JC, Andrade R, Alvarez A. The clinical, neuroradiographic, and endocrinologic profile of patients with bilateral optic nerve hypoplasia. Ophthalmology 1997;104:493-6.

9. Golnik KC. Congenital optic nerve anomalies. Curr Opin Ophthalmol 1998;9:18-26.

10. Bradford GM, Kutschke PJ, Scott WE. Results of amblyopia therapy in eyes with unilateral structural abnormalities. Ophthalmology 1992;99:1616-21.

11. Fu VL, Stager DR, Birch EE. Progression of intermittent, small-angle, and variable esotropia in infancy. Invest $O p h$ thalmol Vis Sci 2007;48:661-4.

12. VanderVeen DK, Coats DK, Dobson V, et al. Prevalence and course of strabismus in the first year of life for infants with prethreshold retinopathy of prematurity: findings from the Early Treatment for Retinopathy of Prematurity study. Arch Ophthalmol 2006;124:766-73.

13. Pediatric Eye Disease Investigator Group. Spontaneous resolution of early-onset esotropia: experience of the Congenital Esotropia Observational Study. Am J Ophthalmol 2002;133:109-18.

14. Brodsky MC. Congenital optic disk anomalies. Surv Ophthalmol 1994;39:89-112.

15. Hoyt CS, Good WV. Do we really understand the difference between optic nerve hypoplasia and atrophy? Eye (Lond) 1992;6:201-4. 